participate in the respiratory movements of the diaphragm. The heart and pericardium are fixed to the roots of the lungs; in orthograde forms the pericardium becomes bound to the diaphragm. ${ }^{3}$ Thus with the evolution of the great anthropoids there came about a new relationship between the diaphragm and roots of the lungs; the central tendon of that muscle became yolked to the roots of the lungs through the pericardium. In the chest of man this relationship is even more intimate than in anthropoids. For the proper aeration of the apical part of man's lungs diaphragmatic movements are essential.

\section{Other Respiratory Changes.}

It would take us too far afield were we to analyse all the intrathoracic changes which mark the evolution of the orthograde posture. The arch of the aorta and the manner in which it gives off its great branches became altered; the human arrangement is linked on to the pronograde type by a series of intermediate stages seen in the great anthropoids. The lung assumed a conical shape, with the wide and hollow diaphragmatic base with which we are familiar in human anatomy. The fissures of the lung become less deep; they have almost disappeared from the lungs of the orang. The apical lobes become larger, the inferior lobes, particularly the parts which fill the hinder and lower recesses of the pleural cavities, smaller. The distribution of the bronchial tree was changed, the apical supply becoming increased.

SUMmary.

It will thus be seen that the evolution of the orthograde posture was attended by a structural revolution in all those parts which are concerned in setting up a tidal flow of air to the lungs. Ribs, vertebrae, sternum, body wall and spinal muscles, diaphragm, pleurae, lungs, and heart underwent a simultaneous and harmonious adaptational transformation. To account for such a structural revolution we must postulate a much more elaborate mechanism controlling developmental processes than any we have knowledge of as yet. Further, it is evident that the square shoulders, the flat back, the wide chest, the cordiform diaphragm, we are familiar with in man's body, are of ancient origin, having been evolved with the appearance of the earliest of orthograde primates.

${ }^{1 T}$ T. Wingate Todd: Anat. Anz., 1912, vol. 41, p. 337. 2 For data relating to these age changes in shoulders and thorax see Wingate Todd relating C. B. Rodes, Zeit. f. Morph. u. An/hrop., 1905, vol. 9, p. 113 (Wintrich's observations are here included); E. Mehnort. Utber tcpographische Alterverd̈derungen des Atmungsapparates, Jenn, 1:01. ${ }_{9}$ For fuller details see Keith, Lancet, 1903, i, p. 6‡2;1904, i, p. 559.

\section{(I)} on

\section{THE NATURE OF ARTERI0-SCLEROSIS.}

\author{
Delivered before the Royal College of Physicians \\ OF LONDON \\ BY \\ GEOFFREY EVANS, M.D.CANTaB., F.R.C.P.Lond., \\ ABgISTANT DIRECTOR, MEDICAL DNIT, AND ASSISTANT PHYBICIAN, \\ ST. BART dULOMEW'S HOSI'ITAL.

\section{LeCtURE III.-CAUSATION OF DIFFUSE HYPER.} \\ PLASTIC SCLEROSIS:
}

The description of diffuse hyperplastic sclerosis formed the subject of the first lecture; the process concerned in its production and the opinion that it was one of inflammation was presented in the second; the matter of its causation remains for consideration.

Since diffuse hyperplastic sclerosis has not been produced experimentally only clinical evidence on causation is avail. able, and before this is discussed the clinical signs of diffuse hyperplastic sclerosis need to be established. The clinical diagnosis is nearly always a matter of inference, since there is rarely the opportunity for microscopical examination of a piece of tissue; though the stuujy of retinal vascular disease holds out the prospect of the accurate diagnosis of diffuse hyperplastic sclerosis during life the correlation of ophthalmoscopic findings with histoiogical lesions is not às yet complete. The clinical picture can be established, however, on the basis of the naked.eye post mortem indications of the presence of the lesion.

\section{Naked-kye Post-mortem Evidence of Diffose Hyperplastid Sclerosis.}

The identity of diffuse hyperplastic sclerosis as an affection of the larger vessels has not been established, hence it can only be recognized by microscopical examination of tissues cut fresh or suitably embedded to retain their fat. Its presence is inferred from the appearance of thickening of the smaller arteries, particularly of the kidneys and spleen, and the vessels are seen to protrude like little quills (in the words of Hilton Fagge) on the cut surface of these organs. When there has been a raised blood pressure during life the walls of the larger arteries such as the splenic and iliac arteries are obviously thickened, and the hypertrophied media shows as small transverse ridges on the inner surface of the vessel wall.

Cardiac hypertrophy, especially affecting the left ventric'e, when not due to disease of the heart and lungs, is termed "idiopathic hypertrophy" for the sake of present convenience, and it is the most important evidence, though not proof, of diffuse hyperplastic sclerosis. Its significance in this respect has been tested by two lines by inquiry. In the first place, an analysis of twenty consecutive years of medical post-mortem records of St. Bartholomew's Hospital ${ }^{1}$ showed that idiopathic cardiac hypertrophy of more than $15 \mathrm{oz}$. weight was significant of cardio-vasculo-renal disease. Thus the records of the great majority of cases with idiopathic hypertrophy showed death due to cerebral haemorrhage, heart failure, or uraemia; or when death was due to other cause the records made reference to arterio-sclerosis or some form of renal sclerosis. The few exc.ptions included a small group of cases of pernicious anaemia (Cabot has also cailed attention to cardiac hypertroplyy in this disease), a few cases of alcoholism, ${ }^{2}$ and obese heart. In the second place, I have found diffuse hyperplastic sclerosis in all cases of idiopathic hypertrophy that I have examined microscopically. The hypertroply of the heart and media of the arteries is to be regarded as a compensatory phenomenon, physiological expressions of increased activity during life. The only essentially pathological sign of diffuse hyperplastic sclerosis is renal sclerosis in one of its several forms - a matter that will be referred to later. In short, the essential post-mortem picture of diffuse hyperplastic sclerosis is limited to the cardio-vasculo-renal system; it consists of left ventricle hypertroply, the appearance of medial thickening, and renal sclerosis. This picture is often dominated by one of the complications or sequelae of vascular disease, such as cerebral haemorrhage, thrombosis, heart failure, or septicaemia; it may also be one aspect of a coincident condition, such as nephritis when death is due to uraemia, and it is often overlooked when death is due to an intercurrent disease such as pneumonia or cancer.

Chinical Picture of Diffuse Hyperplastic Sclerosis,

Post-mortem evidence establishes the clinical diagnosis of diffuse hyperplastic sclerosis on idiopathic cardiac hypertrophy and renal sclerosis. The clinical diagnosis of the former is not without difficulty, since heart disease, particularly myocardial disease, is often difficult to exclude. From a comparison of blood pressures taken during life and heart weight determined after death a close relation has been estab. lished between raised systolic pressure and idiopathic hyper. trophy. The direct relation shown in my first paper on arterio-sclerosis ${ }^{1}$ has not been borne out by a series of 32 cases collected since. The two series are not, however, directly comparable, since the second series was merely compiled from clinical notes and post-mortem records. Other factors than mere increase in blood pressure are concerned in determining the degree of cardiac hypertrophy. Of the 32 cases of idio. pathic hypertiophy that form my second series (heart weight more than $15 \mathrm{oz}$.) the systolic blood pressure during life was $180 \mathrm{~mm} . \mathrm{Hg}$ or over in 84 per cent., and was $170 \mathrm{~mm}$. Hg or over in 94 per cent. 'Ihese cases furnish further evidence of the association of raised blood pressure and idiopathic hypertrophy, which is a!ready generally accepted. In choosing an arbitrary figure of systolic pressure as having a pathological significance it is very necessary to take a conservative line, since the blood pressure is liable to considerable variation in health, $\mathbf{s}$ and in conditions of fatigue, excitement, pain, dyspnoea, and the like, but my experience has been that the systolic pressure does not reach $180 \mathrm{~mm}$. Hg under such conditions; and having regard to the relation of diffuse hyperplastic sclerosis on the one hand, and of a systolic blood pressure of $180 \mathrm{~mm}$. $\mathrm{Hg}$ to idopathio 
cardiac hypertrophy on the other hand, I believe that a systolic pressure of this level is definite clinical evidence of diffuse hyperplastic sclerosis, provided always that the reading is taken under good conditions. In outline my view is that the clinical signs of diffuse hyperplastic sclerosis are idiopathic cardiac hypertrophy (standard being $15 \mathrm{oz}$. weight post mortem) and a raised systolic pressure (180 mm. $\mathbf{H g})$. There is no need to complete the clinical picture, and I will pass on to consider the various diseases in which these two signs are present.

\section{Hyperpiesia.}

Sir Clifford Allbutt' introduced this term to define a group of cases of which the outstanding features are a persistently raised blood pressure with cardiac hypertrophy but without kidney disease; thus far the description applies to a clinical condition, but Sir Clifford Allbutt uses the term to define not merely a condition but a disease - one that may respond to treatment and recover, or alternatively pursue its own course and terminate in death. In this latter event it preserves its identity as a morbid series that does not pass through or into Bright's disease; it is marked by the absence of such classical signs as an earthy complexion, a pronounced secondary anaemia, and also by the condition of the urine, which, though it may contain a few hyaline or granular casts or a small quantity of -albumin, does not in hyperpiesia become pale in colour or low in its specific gravity ; neither does it contain red blood cells or epithelial casts, unless it is complicated by marked venous congestion as a result of heart failure. In view of the relation of cardiac hypertrophy and raised blood pressure to diffuse hyperplastic sclerosis, and since these are the cardinal signs of hyperpiesia, I maintain that the clinical expression of diffuse hyperplastic sclerosis is hyperpiesia.

The connexion between hyperpiesia and its vascular lesion is so close that it is commonly regarded as one of cause and effect. The older view that the vascular lesion is responsible for the hypertension is no longer tenable. 4 The present opinion is that hypertension is the cause of arterio-sclerosis, an opinion that is expressed by Sir Clifford Allbutt in his distinction of a hyperpietic form of arterio-sclerosis. This opinion has received support from the work of Jores, reinforced by his interpretation of his histological findings (Lecture II). It is also supported by the record of cases of hypertension without demonstrable vascular lesions after death ${ }^{467}$; again, the clinical fact that hyperpiesia is a recoverable disease, balanced against the opinion that arterio-sclerosis is a permanent pathological change, a final event from which recovery cannot occur, is urged in the same sense; and lastly, there is the circumstance that blood pressure measurements are taken during life and vascular lesions are studied later after death. Such evidence as this does not bear critical examination. It is not certain that diffuse hyperplastic sclerosis is a permanent pathologica] change, or if it is the body may be able to so adjust itself that the patient recovers from hyperpiesia though his arteriosclerosis persists. Further, to establish a sequence in time between the development of hypertension and diffuse hyperplastic sclerosis does not establish the relation of cause and effect since both may result from the action of a common cause, and the difference in time of their development may depend on the longer time required for the development of a structural lesion as compared with a functional lesion.

Hyperpiesia may be dependent upon a disturbance of vas. cular function due to the activity of a pressor substance in the sense postulated by Dr. Batty Shaw ${ }^{8}$ and Krogh's work ${ }^{9} 10$ on capillariomotor activity throws a new light on the mechanism set in motion at this stage of the disease. A similar problem is present in Raynaud's disease when vaso. constriction is so persistent and severe that gangrene of a distal part results without the occurrence (according to Leo Buerger) of a demonstrable vascular lesion; but in the only case of Raynánd's disease that I have examined there was an extreme degree of arterio-sclerosis in the digital arteries of a finger that had to be amputated for gangrene.

While there is no adequate clinical evidence for the opinion that mechanical strain is a cause of diffuse hyperplastic sclerosis, pathological evidence is contrary to such a view. Jores's views on the subject fall to the ground with the failure of his distinction between regenerative and hyperplastic layers in the thickened intima of diffuse hyperplastic sclerosis. The actual lesion in the arterioles, the endothelial cell proliferation and subsequent fatty degeneration, is not open to explanation in terms of increased blood pressure. Fatty degeneration is an expression of deficient oxidation, and a mechanical strain that shows itself in this weakening of the vessel wall can hardly be responsible at the same time for the increased tissue activity shown by proliferation of the endothelial cells of the perfect form and staining seen in these vessels. Mechanical strain fails equally as a working hypothesis in regard to the distribution of the lesion, for it does not explain the relative incidence of the lesion on the several organs of the body; it is no explanation of its major incidence on the kidneys and spleen, its common presence in the brain, pancreas, liver, and suprarenals, its relative rarity in other organs, and its absence from skeletal muscles and heart. The theory of increased blood pressure fails to account for the development of diffuse hyperplastic sclerosis, and it is concluded from the evidence at present available that the cause of diffuse hyperplastic sclerosis is the same as that of hyperpiesia.

\section{Renal Sclerosis.}

There is some degree of renal sclerosis in all cases of diffuse hyperplastic sclerosis, and there is some vascalar lesion in all cases of renal sclerosis. Apart from polycystic kidneys, tuberculous and obviously syphilitic kidneys, calcu. lous and other forms of pyelonephritis, renal sclerosis is divisible into two main groups according to the distribution of the lesion in the organ.

Group 1: Chronic Nephritis.-The lesion is diffuse.; though not uniform it is widely distributed throughout the organ. The essential lesion is a glomerulitis, shown by pro: liferation of the cells of Bowman's capsule, or at a later stage by adhesion of the parietal and visceral layers of the capsule. The constant lesion, as in all forms of renal sclerosis, is an increase of fibrous connective tissue. Taken as a whole, and in their more chronic forms, these kidneys tend to be associated with diffuse hyperplastic sclerosis and cardiac hyper. trophy. The association of diffuse hyperplastic sclerosis with chronic nephritis is best illustrated by its occurrence in children, of which $I$ have recently published four cases in detail11; from another point of view it is illustrated by statistical evidence of eardiac hypertrophy in its relation to kidney disease (see Ref. 1, p. 248). The lesion in chronic nepbritis is without doubt inflammatory, and its association with diffuse hyperplastic sclerosis has been brought forward as further evidence in favour of the inflammatory nature of the vascular lesion. The vascular lesion may develop to an extreme degree, so that the parent arteries are almost com. pletely obliterated; in other cases of extreme renal disease the vascular lesion nay be relatively slight and limited to the kidneys. Clinically there may be a history of acute nephritis or of oedema of the renal type. The symptoms of which the patient complains are those commonly regarded as uraemic, of which the more important are headache, gastro-intestinal disturbance, and disturbance of vision due to albuminuric retinitis. In well marked cases there is pallor of the face; in severe cases a muddy complexion and secondary anaemia; the urine containg red and white blood corpuscles and cell casts, and at a later stage it is pale in colour and of low specific gravity. Sometimes there are no cells or casts in the urine. Puffiness of the face is common; cardiac oedema is more frequent than renal oedema. Hypertension and cardiac hypertrophy are characteristic but not essential features of the clinical picture.

My interpretation of the association of diffuse hyperplastic sclerosis and chronic nephritis is that both lesions are pro. voked by the same cause or causes, and the solution of the etiology of chronic nephritis will solve the etiology of diffuse hyperplastic sclerosis (see also Ophüls, loc. cit., p. 95). This view has lately received support from the study of the his. tology of war nephritis in the early stages of the disease. Herxheimer ${ }^{12}$ has recorded that the earliest lesion is of the endothelial cells of the glomerular tufts; initially it is limited to occasional loops of occasional glomeruli; soon the whole or the greater part of the tuft is affected and the great majority of all glomeruli are involved. Inflammatory reaction in the cells of Bowman's capsule, the typical lesion in nephritis, is a subsequent erent.

Group 2 includes all kidneys in which the lesion is partial, being limited to wedge shaped areas with their base on the surface and their apex pointing to the cortico-medullary zone, These areas correspond to depressions on the surface of the organ: when they are small the surface is almost smooth (as in the hyperpietic kidney); when they are large and deep the surface is coarsely granular (as in true chronic interstitia) nephritis). The areas consist of fibrous tissue, damaged renal parenchyma, and more or less small cell infiltration. The 
intervening tissue is relatively normal and the parenchyma may be hypertrophied. On account of the presence of normal tissue these kidneys retain their colour (which depends largely on their capillary blood content), in contrast to the kidneys of cluronic nephritis in which the lesion is diffuse and the colour pale.

Subgroup (a): True Chronic Interstitial Nephritis.Histologically there is evidence of active inflammation in the presence of g!omerulitis, similar to that seen in chronic nephritis, and small cell infiltration out of proportion to the degree of glomerular tuft damage. The differential diagnosis of these cases clinically from chronic nephritis may be im. possible. As a general rule they occur after the age of 35 , whereas chronic nephritis is commoner before 35 . The most obvious aspect of the clinical picture is cardio-vascular disease, the symptoms being those of heart failure, or simulating hyperpiesia, or sometimes they are purely vascular, as in gangrene of the toes. The renal aspect of the case, in its symptoms, facies, or urinary findings, is a less prominent feature than the cardio vascular aspect, but to the extent that it is present it is of the same kind as in chronic nephritis. The changes in the fundus oculi are those of arterio-sclerotic retinitis, ${ }^{13}$ in contrast to albuminuric retinitis in chronic nephritis. The most marked cases of diffuse hyperplastic sclerosis are found in this subgroup, and they are associated with the highest systolic blood pressures. These cases with their definitely inflammatory renal lesion are furthcr evidence of the inflammatory nature of diffuse hyperplastic sclerosis and point to a common etiology of it and chronic interstitial nephritis.

Subgroup (b) : True Arterio-sclerotic Kidneys.-This subgroup is further subdivided into two types, according to whether the accompanying vascular lesion is of the type of diffuse hyperplastic sclerosis or senile arterio-sclerosis. ${ }^{1}$ This classification, which I suggested in 1921, seems to hold good in the light of material examined since, and has been recent!y followed by Professor Shaw Dunn. ${ }^{14}$ Chronic nephritis and chronic interstitial nephritis can be similarly subdivided according to the type of the vascular lesion present, but it is not necessary for the present purpose. The essential histological feature of the kidneys in Subgroup $(b)$ is the absence of definite signs of inflammation, apart from the vascular lesion, in these kidneys; the glomeruli undergo hyaline degeneration and fibrosis; there is no proliferation of Bowman's capsule; there is little small cell infiltration, and the degree of small cell infiltration bears some relation to the degree of glomerular tuft damage. Clinically these cases have no renal aspect, and do not suggest the picture of Bright's disease in any of its well recognized forms. The renal lesion is only evident in a trace of albumin in the urine; the urine is of good colour and normal specific gravity. Of the two types included in this subgroup the one in which there is diffuse hyperplastic sclerosis is the more important, for it is associated with raised blood pressure and cardiac hypertrophy, and is hyperpiesia. Microscopically the kidneys are relatively normal in appearance, their surface is smooth, or very finely granular, or they may be marked by scars like old infarcts. On section they are a little firmer than normal, and the vescels are unduly prominent. I suggest the name "hyperpietic kidney" for this type of renal sc!erosis, and its significance in respect to the etiology of diffuse hyperplastic sclerosis has been already discussed under hyperpiesia.

This slight review of the relation of nephritis and renal sclerosis to diffuse hyperplastic sclerosis is sufficient to establish the intimate relation of the two conditions. It emphasizes the paramount importance of vascular lesions in renal disease, and it is this association that will, I think, ultimately lead to the solution of the causation of arterio-sclerosis. It is possible to assume, as have Aschoff, Gaskell, and others, that the vascular lesion is the cause of the renal lesion in my seco nd group of renal sclerosis- the arterio-sclerotic groupa point of view that involves Gull and Sutton's conception of diffuse hyperplastic sclerosis as an independent disease of the vascular system. I hesitate to accept this view, because it cannot hold good for the relation of diffuse hyperplastic sclerosis to chronic nephritis and chronic interstitial nephritis. It is possible that the inflammatory changes in the latter are due to secondary infection; as has been suggested by Volhard and Falr, ${ }^{15}$ a suggestion which is as difficult to prove as it is to refute. There is less objection to the view that the vascular disease is primary and the renal lesion a secondary atrophy in the hyperpietic and senile forms of arterio-sclerotic kidneys-Subgroup $(b)$. At the same time this gives the vasculature an identity of its own, whereas it is better regarded as merely part of the cardio-vasculo-renal system.

The basic proposition that I have attempted to present in this outline of the relation of hyperpiesia and renal sclerosis to diffuse hyperplastic sclerosis is that the causation of the vascu'ar lesion is the same as the causation of hyperpiesia and renal sclerosis. The etiology of this condition, so far as it is known at present, is due to the action of bacterial toxins, and both the character of the vascular lesion in diffuse hyperplastic sclerosis and the distribution of the lesion in the vascular tree is compatible with the view that it is caused by a circulating toxin. Thus, with regard to the former, the character of the lesion is inflammatory; and with rrgard to the latter, it is known clinically from the distribution of amploid disease and experimentally from the work of Welch and Flexner ${ }^{16}$ and others, that a circulating bacterial toxin can readily cause both an unequal and a focal lesion of arterial wails.

The Relation of other Forms of Arterio-sclerosis to Diffuse Hyperplastic Sclerosis.

\section{Nodular Sclerosis.}

This lesion is like diffuse hyperplastic sclerosis in that the initial cliange is in the intima and in that the early stages slow an intimal thickening due to proliferation of superticial cells, which Klotzin regards as either of endothelial or connective tissue origin. The intimal thickening is permeated by elastic fibrils at a later stage, and fatty degeucration occurs subsequently, perhaps as a result of deprivation of oxygen. Klotz and Manning ${ }^{18}$ have made a minute study of the fatty streaks and spots that are chiefly found in the aorta and in the main vessels arising from it. They are of the opinion that these lesions may develop into nodular sclerosis and atheroma, an opinion shared by Jores and others; they say of these lesions that

"there is every indication that the production of tissue in the intima is the result of a direct irritation of that tissue by the presence of infection or toxins or of the stimulation by the products of a primary degeneration in that layer."

There is considerable evidence in favour of the view that these lesions, as well as those which are doubtless nodular sclerosis, are due to the effect of bacterial toxins. Klotz suggests that they are of an inflammatory nature, and he controverts Thoma's theory that the intimal thickening is compensatory to medial degeneration. From the information at prosent available it would seem that nodular sclerosis, in the nature of its lesion and in the site of its earliest appearance in the vessel wall, resembles diffuse hyperplastio sclerosis, while in other circumstances Thoma's theory may hold good-that it is a compensatory thickening secondary to an initial weakening of the media.

\section{Senile Arterio-sclerosis.}

This form of arterio-sclerosis is primarily a medial degeneration, beginning with fatty degeneration and atrophy of the muscle fibres, and affecting the larger and smaller vessels. ${ }^{19}$ Secondary to these changes occur replacement fibrosis and deposit of lime salts in the media. It is commonly accompanied by thickening of the intima, and nodular arterio. sclerosis is generally present in some degree. It differs from diffuse hyperplastic sclerosis in its clinical expression; age is more definitely a factor in its causation, and it is not accom. panied by the cardiac hypertrophy and raised blood pressure of diffuse hyperplastic sclerosis.

Senile arterio-sclerosis is as diffuse in its distribution as diffuse hyperplastic sclerosis, but it differs from it both in respect of the site of the earliest lesion and in the character of this lesion. It is possible, therefore, that its causation is different; vascular lesions that have been produced experimentally, such as those caused by repeated adrenaline injections, ${ }^{20}$ also by experiments (Klotz) in which arterio-sclerosis of the aorta was produced in rabbits by suspending them by their hind legs for two or three minutes daily for many days in succession, are of the nature of medial degeneration with calcification. Again, clinical conditions, such as mitral stenosis, that lead to an increased pressure in the pulmonary circulation sometimes (though not always) lead to arteriosclerosis of the pulmonary artery in which degenerative changes are the prominent feature.

There is both clinical and experimental evidence, therefore, that mechanical and toxic causes may be responsible for senile arterio-sclerosis. I'horel's $\mathrm{x}^{21}$ opinion on the mechanical causation of arterio-sclerosis expresses as well as any other 
the generally accepted view. After reviewing the conditions in which an increased blood pressure is generally regarded as responsible for the vascular lesion, he remarks that "the factor of increased content of metabolites in the blood is also involved," and he expresses the opinion that an increased blood pressure cannot be excluded from the etiology of arterio-sclerosis, and that "it is without doubt an accessory factor in the production of arterio-sclerosis provoked by other causes." For a full discussion of this subject reference should be made to Allbutt, Andrewes, Thorel, MacCallum, and Moschcowitz.

Whether, however, a degenerative or proliferative lesion results from the action of a toxin on the vessel wall, may depend on the one hand on the nature of the pathogenic agent. On the other hand, the power of resistance of vascular tissues to the pathogenic agent must be an important determining factor, and it is possible that whether diffuse hyperplastic sclerosis or senile arterio-sclerosis result depends rather on the vitality of the tissues affected than on the nature of the pathogenic agent. There is some evidence in farour of this view, and factors such as age and inheritance find their due place in the tangle of causation in the extent to which they undermine and modify the reaction of vascular tissues to insult. Thus there is no reason to believe that age in itself is the cause of arterio-sclerosis, and Ophüls ${ }^{22}$ has shown that the curve of incidence of arterio-sclerosis does not correspond with what would be expected if the lesion were the direct result of it and of the senile deterioration associated with it.

\section{Mönckeberg's Sclerosis.}

Mönckeberg's sclerosis illustrates this aspect of the problem from another point of view. The form of sclerosis that he describes is often included in the senile form of arterio-sclerosis; it does indeed often occur in old age, and it is like senile arterio-sclerosis in the fact that it is apparently a primary affection of the media and is characterized by calcification. In a sense it is more senile than senile arterio. sclerosis, for the intima often fails to proliferate, and when it occurs it is often late in the evolution of the lesion as a tardy process of repair. But it is not age that has weakened the vessel walls and prevented their reaction to insult-it is disease, not local but general; thus of the 12 pure cases described in Mönckeberg's original paper, 23 died of cancer, 4 died of phthisis, 1 of advanced morbus cordis, and 3 of cachexia due to chronic infection. I think it is here that diabetes mellitus sometimes finds its place in the etiology of arterio-sclerosis.

SUMmary.

The study of the pathology of arterio-sclerosis has impressed me with the vivid picture of reaction in tissues to insult, response to injury, and repair of damage done. Great students of pathology such as Virchow interpreted the lesion in terms of inflammation, Thoma in terms of involu. tione and Jores in terms of growth. These men, at least, have failed to grasp the cloven hoof of decadence and decay. It is asking too much to expect a restitutio ad integrum in arterio-sclerosis, and, as in tuberculosis of the lung, we may well be content with a lesion that heals by calcification and fibrosis. The prospect of recovery in vascular tissue is indeed better than it is in the parenchyma of the lung; new vessels are formed more easily than new. paren. chyma. Louis Gross ${ }^{24}$ has demonstrated the formation of new ressels in the kidney of renal sclerosis, and the rapidity of their formation is strikingly illustrated in thromboangeitis obliterans, where new ressels may be formed in the lumen of the affected artery before the original process of disease has subsided, so that the new ressels may themselves be affected by the original process of disease. Again, it is remarkable evidence of the healing power of vessels that aneurysm of the aorta is rarely due to arterio-sclerosis even when present in a severe degree. There is a big field for inquiry in clinical medicine where evidence of the healing of arterio-sclerosis may be collected, and yet before the gate into this field is opened the clinical diagnosis of arterio-sclerosis needs a closer approach.

As the problem of arterio-sclerosis is contained in its definition, I will end by stating the problem as I have tried to outline it :

Axterio-sclerosis is a lesion characterized by a pathological thickening of arterial coats, particularly of the intima; inflammatory reaction is of first importance; degenerative changes are an essential part of the picture; hyperplastic and involutionary processes play a part; no unit factor is responsible for its cauation. Its development in its extent and form depends on a balance between the action of toxic agents and the power of resistance of the vessel wall to their attack.

In conclusion, it is a real pleasure to acknowledge the help and advice that I have received from Sir Frederick Andrewes, and the constant assistance kindly given by Sir Bernard Spilsbury.

1 Evans, Geoffrey : Quart. Journ. Med., Oxford, 1921, 14, 215. ${ }^{2}$ Fishor, Theodore: Guy's Hosp. Reports, 1894, 51, 97. 'Oliver, George: Studies in Blood Pressure, third edition, 1916. 116. A Allbutt, Sir Clifford: Diseases of the Arteries and Angina Pectoris, 19i5. SMacCellum, W. G.: Physiol. Reviews, 1922, 2, 70. 6 Strouse and Kelman : Arch. Int. Med., 1923, 31, 151. 7 Moschcowitz, Fli: Journ. Amer. Med. As 800., 1922, 79, 1196:: 1905, 59, 319. 8 Shaw, H. Batty : Hyperpiesia and Hyperpiesis, Oxford Med. Pub., 1922. 9 Krogh, A.: Journ. Physiol., 1919, 52, 457. 10 Krogh, Harrop, and Rehberg: Ibid., 1922, 56, 179. 11 Evans, Geoffrey: Quart. Journ. Med.. Oxford, 1922, 16, 33. 12 Herxheimer, G.: Handb. d. Arztl. Erfahr. im Weltkriege, 1921, 8. ${ }^{13}$ Moore, R. Foster: Quart. Journ. Med. Oxford, 1916, 10, 30. 14 Dunn, John S.: BRrrish MEDTCAL JoURNAL, 1922, ii. 1166 , 15 Volhard and Fabr: Die Brightsche Krankheit, J. Bpringer, 1914. is Welch and Flexner: johns Hopkins Hosp. Bull. 1892, 3, 17. 17 Klotz, Oskar: Journ. Exp. Med. 1910, 12, 707. 18 Klotz and Manning: Journ. Path. and Pact.. 1911, 16, 21i. 19 Klotz, O.: Journ. Exp. Med., 1906, 8. 322. 20 Josue: Presse Med., 1903, 11, 798; O. R. Soc. de Biologie, 1905, 59, 319. 21 Thorel, C. : Lubarsch-Ostertag, $1915,1,182 .{ }^{22}$ Ophtils, William: Stanford Univ. Pub.
Med. Sci., 1921, 1, 5. 23 Mönckeberg, J. G.: Virohr.w's Archiv., 1903, 171, 141.

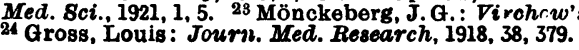

\section{ON ANKYLOSTOME INFESTATION : THE CHANGING PROBLEM.}

BY

CLAYTON LANE, M.D., LieUt.-CoL: I.M.S.(RET.).

The present time seems particularly opportune to call atten. tion to certain aspects of the hookworm problem which threaten to cause a division of opinion and procedure among those whose common desire is its solution.

It is probably undisputed that the aggregate of ankylostome infestation is enormous, hundreds of millions of persons being infested, that the effects' of comparatively light infestations are harmful, and that an active hookworm campaign is essential. Beyond this point opinion is imperfectly orystal. lized. There is, for example, a weighty body which holds that the problem of ankylostomiasis is so great that it. must be attacked along lines admittedly unscientific and deliber. a'ely rule of thumb. Their case is a reasoned one and can be put, I think quite fairly, thus :

1. The microscope fails to detect with certainty light hookworm infestations ; so that, relying upon it, the sanitarian, intent upon producing complete disinfestation, will find nothing but disappointment.1

2. The light infestations, those with less than ten worms, missed by the microscope, do not, however, damage their host; so that the fact that the microscope misses such infestations is actually an advantage.2

3. Accordingly the right way to attack the hookworm problem in any locality where infestation is shown to be considerable is to treat all persons indiscriminately, without attempt at individual disonosis, with two treatments of oil of chenopodium 8

4. In this way 93 to 97 per cent. of the worms will be removed and all infestation material to the host will be eliminated.1 3

5. At the same time the soil will cease to be seriously infected, and reinfection of man will remain at the point where the resulting human infection will be immaterial. ${ }^{3}$

6. So that, in view of the inadequacy of microscopic diagnosis, and the practical adequacy of treatment, it is the duty of the sanitarian, faced with this huge problem, to concentrate upon the heavy cases which matter and disregard the light ones which do not.

Microscopic Detection of Ova.

Whether infestation be light or heavy, the microscopic detection of ora in the stool is manifestly impossible if the worms be not ovipositing-that is to say, if only males be present, or if the females be too young, too old, or too ill to lay. If ora be present in the stool can they infallibly be detected there by the microscope, or is it the case, as stated in the fifth annual report of the Rockefeller Foundation, International Health Board, that microscopio technique fails to detect 50 per cent. of light infestations? The answer to this lies in some sixteen months' work of mine, financed first by the International Health Board and later by the Royal Society.' This work starts from the admitted position that hookworm eggs may be so sparse in a stool that the West Indian technique is fully justified. This requires that before a negative diagnosis is made nine faecal smears on $3 \times 2$ in. 